\title{
Das Geschäftsmodell Data Facilitator im Consulting
}

\author{
Volker Nissen
}

Eingegangen: 15. Dezember 2020 / Angenommen: 17. März 2021 / Online publiziert: 9. April 2021

(C) Der/die Autor(en) 2021

Zusammenfassung Mit der Vervielfachung des Datenvolumens und der raschen Weiterentwicklung entsprechender Analysemethoden und Werkzeuge gibt es immer mehr Unternehmen, insbesondere aus dem kleineren Mittelstand, die aufgrund mangelnder Ressourcen und Fähigkeiten nicht in der Lage sind, ihre Datenschätze zu heben und daraus gute Entscheidungen im Hinblick auf ihre Produkte, Prozesse und Geschäftsmodelle abzuleiten. Vor diesem Hintergrund wird im vorliegenden Beitrag ein Data Facilitator Geschäftsmodell für Consultingunternehmen vorgeschlagen. Um nicht im Wettbewerb zurückzufallen, lagern hierin Unternehmen Aufgaben der Datenaufbereitung, Analyse und Interpretation an ein Beratungsunternehmen aus, das sich auf solche Themen spezialisiert hat. Berater erhalten dabei Zugriff auf kritische Datenströme des Kunden, die dann auf regelmäßiger Basis fachkundig, unter Einsatz moderner Analyseinstrumente untersucht werden. Es geht darum, frühzeitig „schwache Signale“ für zukünftige kritische Entwicklungen beim Kunden zu erkennen oder Ideen für geschäftliche Verbesserungen zu entwickeln. Dabei werden die Ergebnisse dem Kunden nicht kommentarlos übermittelt, sondern Auffälligkeiten von den Beratern unter Einbringung ihres Fachwissens mit den Kunden diskutiert und interpretiert. Der Kunde trifft dann gegebenenfalls Entscheidungen auf Basis der vorliegenden Datenlage. Da diese Dienstleistung fortlaufend erbracht wird, sind hierfür neue Bezahlmodelle im Consulting sinnvoll, wie z. B. eine monatliche Pauschale. Für eine erfolgreiche Umsetzung des Geschäftsmodells sind in der Praxis eine Reihe von Problemen zu bewältigen, worauf im Beitrag ebenfalls eingegangen wird.

Schlüsselwörter Unternehmensberatung · Big Data Geschäftsmodell · Data Facilitator · Virtualisierung · Abonnement-Bezahlmodell

\footnotetext{
V. Nissen $(\bowtie)$

FG WI für Dienstleistungen, TU Ilmenau, PF 1005 65, 98684 Ilmenau, Deutschland

E-Mail: volker.nissen@tu-ilmenau.de
} 


\section{The Business Model Data Facilitator in Consulting}

Abstract With the strong rise of data volume and the rapid development of corresponding analysis methods and tools, there are more and more companies, especially from the SME sector, that are, due to a lack of resources and skills, not able to leverage their data treasures and derive good decisions from them with regard to their products, processes and business models. Against this background, this paper proposes a data facilitator business model for consulting firms. In order not to fall behind the competition, companies outsource data preparation, analysis and interpretation tasks to a consulting firm that specialises in such topics. Consultants are given access to critical data streams of the client, which are then expertly examined on a regular basis using modern analytical tools. The aim is to identify "weak signals" for future critical developments at the client at an early stage or to develop ideas for business improvements. The results are not communicated to the client without comment, but conspicuous features are discussed and interpreted by the consultants together with the client, using their specialist knowledge. The client then makes decisions, if necessary, on the basis of the available data. Since this service is provided on an ongoing basis, new payment models in consulting make sense, such as a monthly flat rate. For a successful implementation of the business model, a number of problems have to be overcome in practice, which are also discussed in the article.

Keywords Consulting · Big Data Business Model · Data Facilitator · Virtualisation · Flat-Fee Payment Model

\section{Motivation und Forschungsgegenstand}

Das Aufkommen von „Big Data“ bietet Unternehmen ungeahnte Möglichkeiten, Wettbewerbsvorteile auf- und auszubauen. Um das strategische Geschäftspotenzial von Big Data zu nutzen, haben viele Unternehmen damit begonnen, ihre Geschäftsmodelle weiter zu entwickeln oder gänzlich zu erneuern (Wiener et al. 2020). Auch für die Branche der Unternehmensberatung birgt diese Thematik gewaltige Potenziale. Unternehmensberatung (Consulting) wird hier verstanden als professionelle Dienstleistung, die durch eine oder mehrere, fachlich dazu befähigte und von den beratenen Kunden hierarchisch unabhängige Person(en) zeitlich befristet sowie meist gegen Entgelt erbracht wird. Sie hat zum Ziel, betriebswirtschaftliche Probleme des beauftragenden Unternehmens interaktiv mit den Kunden zu definieren, strukturieren und analysieren, sowie Problemlösungen zu erarbeiten und auf Wunsch ihre Umsetzung gemeinsam mit Vertretern des Kunden zu planen und im Unternehmen zu realisieren (Nissen 2007).

Beratung ist traditionell ein personalintensives Geschäft. Dabei bearbeiten Berater gemeinsam mit Klienten interaktiv (Face-to-Face) unternehmensrelevante Problemstellungen. Inzwischen beginnen jedoch die gleichen disruptiven Kräfte, die bereits die Geschäftsmodelle anderer Branchen verändert haben, den Beratungssektor zu transformieren (Parakala 2015). Unter anderem betreten neue Wettbewerber mit innovativen Geschäftsmodellen und technologiegetriebenen Beratungsansätzen den 
Markt. Viele von ihnen liefern innovative Lösungen für einen bestimmten Schritt in der Wertschöpfungskette des Consulting, wie z. B. die automatisierte Datenanalyse und -interpretation. Technologien wie maschinelles Lernen und moderne analytische Applikationen, Process und Data Mining halten auch im Consulting Einzug. Es ist zu erwarten, dass ein Großteil des zukünftigen Wachstums im Consulting an diejenigen geht, die erfolgreich technologiebasierte Beratungsansätze und Liefermodelle schaffen, welche die Kundenanforderungen besser und zu geringeren Kosten erfüllen. Beratungsmanager sollten daher ihr Leistungsportfolio kritisch überprüfen und das traditionelle, kostspielige Beratungsmodell überdenken. Letztlich geht es darum, bessere Wege zu finden, das Geschäft ihrer Kunden agil und wertschöpfend zu unterstützen.

Berater müssen zunehmend „datengetrieben“werden. Einerseits können sie Daten und analytische Kompetenzen stärker in ihre Dienstleistungen integrieren und damit ihren Mehrwert für die Klienten verbessern. Andererseits kann sich das Geschäftsmodell von Beratungsanbietern signifikant verändern, wenn Daten zur Virtualisierung der Leistungserbringung (Nissen 2018) genutzt werden. Der Übergang eines physischen Prozesses zu einem virtuellen Prozess wird als Prozessvirtualisierung bezeichnet (Overby 2008). Ziel der Virtualisierung im Consulting ist es, die persönliche Interaktion zwischen Berater und Klienten durch den Einsatz von Informations- und Kommunikationstechnologien sinnvoll zu reduzieren. Durch teilweise oder vollständige Automatisierung von Beratungsleistungen, auch mittels digitaler Plattformen, wird die klassisch schlechte Skalierung im Consulting-Geschäft verbessert. Virtualisierung kann als Strategie zur digitalen Transformation des Beratungsgeschäfts bezeichnet werden. Virtuelle Services ergänzen traditionelle Beratungsleistungen, um das Leistungsportfolio zu optimieren und erweitern. Virtualisierung erschließt auch potenziell neue Märkte, die sonst geografisch nicht erreichbar oder für traditionelle Beratungsformen ungeeignet wären (Nissen 2018).

Das nachfolgend zunächst nur kurz dargestellte Geschäftsmodell entspricht diesem Ansatz, durch Digitalisierung und Vernetzung in der Beratung eine neue Form der Interaktion mit den Kunden zu erreichen. Es lässt sich vorrangig in die Big Data Business Modell (BDBM)-Kategorie „Data Facilitator“ nach Schröder (2016) sowie Wiener et al. (2020) einordnen. Anbieter mit diesem Geschäftsmodell sind dadurch gekennzeichnet, dass sie die Versorgung von Datennutzern und -anbietern mit Big-Data-Infrastrukturlösungen sowie mit Big-Data-bezogenen Dienstleistungen übernehmen. Dabei konzentrieren wir uns vorrangig auf die Übernahme komplexer Analytik-Leistungen durch den Anbieter im Sinne eines Outsourcing. Die Darstellung des Geschäftsmodells orientiert sich an der von Al Debei und Avison (2010) vorgeschlagenen folgenden Strukturierung:

Value Proposition: Das Wertversprechen beschreibt die Marktangebote (Produkte und/oder Dienstleistungen) einer Organisation sowie deren Interaktionen mit der anvisierten Kundengruppe.

Value Architecture: Die Wertarchitektur bezieht sich auf die Kernressourcen und -fähigkeiten einer Organisation sowie deren Konfigurationen, die für die Erstellung der Marktangebote erforderlich sind.

Value Network: Das Wertnetzwerk stellt die Interaktionen und Beziehungen einer Organisation mit wichtigen externen Partnern und anderen Stakeholdern dar. 
Value Finance: Diese erfasst die ökonomische Konfiguration einer Organisation, einschließlich ihrer Kostenstrukturen, Preismethoden und Ertragsströme.

Hintergrund der Überlegungen ist, dass noch zu wenige Unternehmen sich für ihre Entscheidungen alle verfügbaren Daten zunutze machen. Dies bestätigte erst kürzlich wieder eine Umfrage der Unternehmensberatung Sopra Steria Consulting (2018). Während Daten im Überfluss vorhanden sind, ist es die Fähigkeit, sie zu integrieren, zu analysieren und intelligent für die Entscheidungsfindung zu nutzen, die den Wettbewerbsunterschied ausmacht. Dies ist insbesondere für kleinere Unternehmen kaum umsetzbar, da sie weder die Konzepte und Methoden beherrschen (Kobek 2020) noch über die relevanten IT-Werkzeuge verfügen. Andererseits sind aufgrund der großen Wirkung und umfassenden Reichweite des digitalen Wandels die Verantwortlichen in Unternehmen dazu angehalten, diesen aktiv zu gestalten, um mögliche Nachteile zu reduzieren und sich bietende Chancen zu nutzen (Fürst 2019).

In dieser Situation kann der zwischenbetriebliche Datenaustausch eine kritische Rolle bei der Schaffung von Werten aus Big Data spielen (Wiener et al. 2020). Dabei übernimmt ein Beratungshaus als Outsourcing-Dienstleister die genannten Aufgaben weitgehend, wobei der Kunde den Zugriff auf kritische Datenströme ermöglicht. So kann die Unternehmensberatung potenziell alle datengenerierenden Bereiche fachkundig analysieren, aufarbeiten und anschließend die Auffälligkeiten gemeinsam mit dem Kunden besprechen und interpretieren. Im Ergebnis werden sowohl Schwachstellen offengelegt als auch Marktchancen oder andere Potenziale aufgezeigt. Die Beratung agiert somit als Absicherung für die Klienten. Es soll möglichst sichergestellt werden, dass nichts Wichtiges in deren relevanten Datenströmen übersehen wird. Für diesen Service zahlen die Klienten eine regelmäßige Gebühr (Beratung im Abonnement).

Dieses hier kurz skizzierte Geschäftsmodell wird im folgenden Abschnitt entlang der Dimension von Al Debei und Avison (2010) genauer entwickelt. Die dabei eingesetzte Forschungsmethode beruht auf einer argumentativ-deduktiven Analyse (Wilde und Heß 2007) vor dem Hintergrund tiefer Kenntnisse des Beratungsmarktes. Im dritten Abschnitt geht es dann um mögliche Problembereiche und Gestaltungsmöglichkeiten in der praktischen Umsetzung. Dabei kann die Unternehmensberatung von den Erfahrungen anderer Branchen, wie der Logistik und dem Maschinenbau, lernen, in denen Outsourcing und datentechnische Integration seit langem existieren und Data Facilitator Geschäftsmodelle schon heute eine zentrale Rolle spielen. Ein kurzes Fazit schließt den Beitrag ab.

\section{Das Geschäftmodell Data Facilitator im Consulting}

\subsection{Value Proposition}

Im Big-Data-Geschäftsmodell Data Facilitator (Schröder 2016) konzentriert sich die Wertschöpfung auf die Bereitstellung von Big-Data-bezogenen Beratungsdienstleistungen und die Bereitstellung von ausgelagerten Big-Data-Analysediensten. In einer Situation, in der ein Klient nicht in der Lage ist, relevante Daten selbst aufzubereiten, 
zu analysieren und interpretieren, kann dieses Unternehmen solche Aufgaben und die Überwachung interner oder relevanter externer Datenströme an ein Beratungshaus auslagern (Outsourcing), das sich darauf spezialisiert hat. Es geht darum, frühzeitig „schwache Signale“ (Ansoff 1975) zukünftiger kritischer Themen beim Kunden zu erkennen. Dabei kann es sich beispielsweise um sich abzeichnende Marktverschiebungen, Mängel in Geschäftsprozessen, IT-Probleme etc. handeln. Ebenso könnten sich aus der Analyse der Daten Ideen für Geschäftsverbesserungen in einer Form von Open Innovation ergeben. Berater machen dann ihre Klienten frühzeitig auf Sachverhalte aufmerksam, die Maßnahmen des Managements erfordern, um wesentliche Probleme zu verhindern oder eine vorteilhafte Situation zu nutzen. So soll sichergestellt werden, dass die Kundenseite keine kritischen Entwicklungen übersieht, die in den verfügbaren Datenbeständen erkennbar gewesen wären. Ähnlich wie sich kleine Online Shops das Fulfillment als „fertige“ externe Dienstleistung zukaufen oder Handelsunternehmen die Bestandsverantwortung für bestimmte Artikel im Logistik-Szenario Vendor-Managed-Inventory (VMI) an ihre Lieferanten übertragen und diesen dafür sensible interne Daten bereitstellen (Waller et al. 1999), würde sich ein kleiner Mittelständler nicht um die Themen Data Engineering, Data Mining \& Analytics kümmern, sondern diese als externe Dienstleistung zukaufen.

Es muss betont werden, dass im vorliegenden Geschäftsmodell letztlich immer der Klient die Verantwortung für aus Daten abgeleitete Managemententscheidungen hat. Dies ist nichts Neues - auch in jedem klassischen Beratungsprojekt trifft stets der Kunde die Entscheidungen. Dies ist wichtig, gerade im Hinblick auf Haftungsfragen bei möglichen Fehlentscheidungen. Die Leistungserbringung wird im Rahmen eines Dienstvertrages und nicht eines Werkvertrages erfolgen. Das Wertversprechen der Berater bezieht sich insofern vor allem darauf, die vom Kunden bereitgestellten Datenströme im Hinblick auf die genannten Zielsetzungen aufzubereiten, gegebenenfalls zu integrieren (auch mit internen Daten der Beratung oder weiteren externen Datenquellen) sowie mit adäquaten Methoden und Werkzeugen zu analysieren. Anschließend werden die Klienten ausführlich und regelmäßig über die Ergebnisse der Analysen informiert. Dabei genügt es nicht, die Ergebnisse den Klienten unkommentiert zur Verfügung zu stellen. Vielmehr sind insbesondere die hier erwähnten wenig technologie-erprobten kleineren Mittelständlern, vor dem Hintergrund weiter ansteigender Datenmengen und Datenvielfalt, auf entsprechende Hilfen durch Berater angewiesen. Für die gemeinsame Interpretation der Ergebnisse und beim Ziehen von Schlussfolgerungen ist auch weiterhin eine persönliche Interaktion von Berater und Klient, vor Ort oder online, notwendig. Insofern kombiniert das Data Facilitator Geschäftsmodell virtuelle und klassische Formen der Unternehmensberatung.

\subsection{Value Architecture}

Für das Geschäftsmodell Data Facilitator müssen Beratungshäuser mehrere Voraussetzungen im Sinne kritischer Ressourcen mitbringen. Zum einen ist eine meist über Jahre etablierte vertrauensvolle Beziehung zu den betreffenden Kunden notwendig. Andernfalls ist kaum zu erwarten, dass für die Wettbewerbsposition des Kunden relevante und somit sensible Daten auf regelmäßiger Basis dem Beratungshaus, in der Regel per Fernzugriff (denkbar ist auch eine Vor-Ort-Analyse beim Klienten) 
zur Analyse bereitgestellt werden. Außerdem erleichtert es die Kenntnis der jeweils individuellen Situation beim Kunden für das Beratungsunternehmen, die Daten im richtigen Kontext einzuordnen und Fehlinterpretationen zu vermeiden.

Zum anderen sind sehr anspruchsvolle analytische Fähigkeiten auf Seiten der Berater notwendig. Starke Fähigkeiten in fortgeschrittener Analytik, einschließlich Data- und Process-Mining, Statistik und künstlicher Intelligenz sind unabdingbar. Darüber hinaus muss ein Data Facilitator Zugang zu aktuellen unterstützenden ITWerkzeugen (bereitgestellt möglicherweise von Drittanbietern als SaaS-Lösung) haben und über das nötige Wissen verfügen, um diese in den verschiedenen Geschäftskontexten der Klienten adäquat einzusetzen. Branchenwissen und Erfahrung aus früheren Beratungsprojekten zu ähnlichen Themen erscheint dabei unabdingbar, um den Besonderheiten der jeweiligen Unternehmenssituation angemessen Rechnung zu tragen.

Ein Teil der analytischen Fähigkeiten könnte sogar automatisch in Form von wissensintensiven Software-Tools bereitgestellt werden, die vom Beratungsunternehmen beim Klienten installiert und mit relevanten Datenquellen wie ERP-Systemen verbunden werden. Diese Tools sammeln und analysieren dann selbstständig Daten aus dem Kundenunternehmen und informieren den „Kontrollraum“ beim Beratungsanbieter über Auffälligkeiten in den Daten. Das Geschäftsmodell lässt sich gut mit fokussierter Datenbereitstellung kombinieren, da Beratungsunternehmen oft Marktdaten aus internen Untersuchungen oder Kundendaten aus früheren Projekten sammeln. Diese Daten können anonymisiert, aggregiert und gebündelt werden, um Daten von aktuellen Kunden zu vergleichen, Branchen- und Marktentwicklungen besser zu verstehen und generell die Analyse für den jeweiligen Klienten wertvoller zu machen, indem die Datenbasis erweitert wird.

Für ein Beratungshaus, das in der geschilderten Weise viele Klienten bedient und dabei große Mengen unterschiedlicher Daten analysiert, ist es notwendig, eine effiziente Integration zu einer Vielzahl heterogener Datenquellen auf der Kundenseite

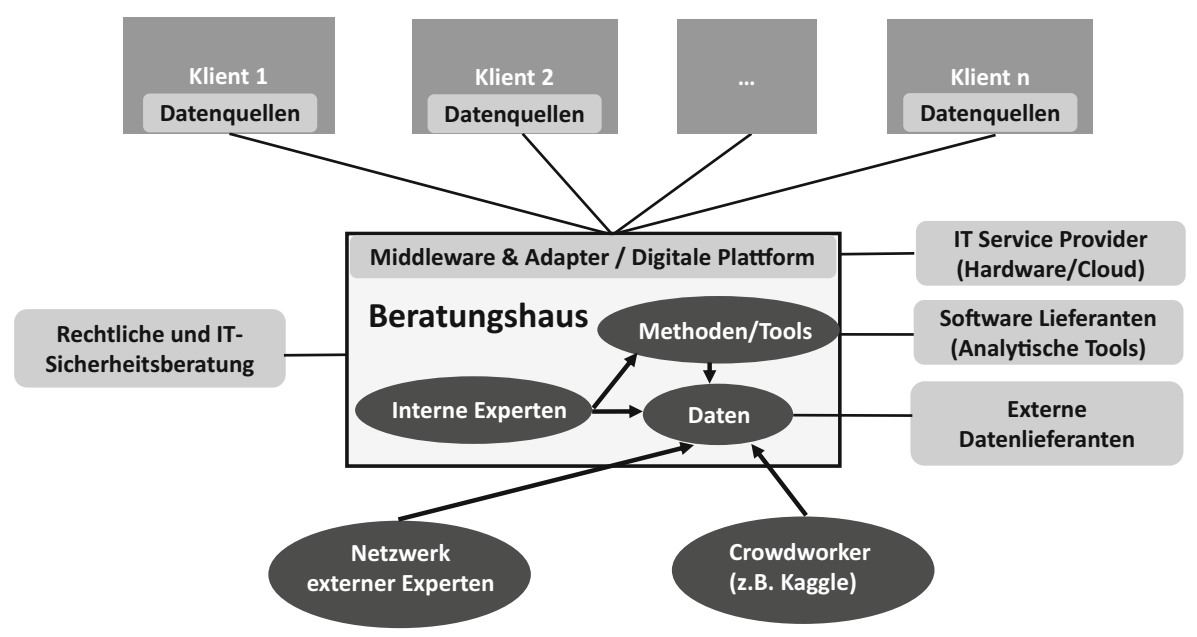

Abb. 1 Komponenten im Netzwerk des Data Facilitator Geschäftsmodells für Beratungsunternehmen 
zu schaffen (Abb. 1). Dies kann über geeignete Datenformate bzw. eine Middleware auf einer digitalen Plattform erreicht werden, wo zusätzliche Klienten oder andere externe Stakeholder mit vertretbarem Aufwand hinzugefügt werden können. Dabei ist es sinnvoll, die Daten der Klienten für Analysen dauerhaft im Rahmen eines Data Warehouse zu speichern.

Welche Lösung sich im Fall des Beratungsunternehmens zur datentechnischen Integration einer Vielzahl von Klienten anbietet, hängt von verschiedenen Faktoren $\mathrm{ab}$, beispielsweise davon wie groß die regelmäßigen Datenmengen sind, ob die Daten in Echtzeit bzw. aus den produktiven Systemen abgerufen werden sollen oder durch den Klienten vorher z.B. exportiert und separat bereitgestellt werden (im einfachsten Fall als CSV-Files). Gerade in den Bereichen Systeminteroperabilität und Datenaustausch ist es dringend erforderlich, sich an den relevanten Standards zu orientieren. Auch ist eine hinreichende Skalierbarkeit der IT-Infrastruktur wichtig, um mit einer schnellen Verbreiterung der Kundenbasis, wie sie plattformbasierte Geschäftsmodelle anstreben, Schritt halten zu können. So lassen sich zumindest einige der von Wiener et al. (2020) ermittelten typischen Herausforderungen von Big Data bezogenen Geschäftsmodellen erfolgreich meistern.

Für den hier unterstellten Anwendungsfall eignen sich prinzipiell die Cloud-Angebote von Amazon AWS, Google Cloud oder Microsoft Azure, wobei sich das nachfolgende Vorgehen auch mit einer unternehmensinternen Cloud der Beratung lösen ließe. Ein praktikabler Ansatz zur Integration wäre, für jeden Kunden eine eigene Daten-Pipeline zu entwickeln. Der Kunde würde seine Daten in beliebiger Form (Excel, CSV, JSON, andere Exportformate) in die Cloud laden, wo sie ab dem Zeitpunkt im Zugriff der Beratung liegen. Beispielsweise als Export der zu analysierenden ERP/CRM Daten oder Excel-Files. Alternativ wären aber auch EchtzeitStreams möglich oder gegebenenfalls auch die direkte Anbindung an die Datenbank des Kunden. Je nach Datentyp und Umfang der Datenmenge sind hier verschiedene Ansätze erforderlich. Anschließend werden die Daten so aufbereitet, dass sie für Analyse-Software des Beraters geeignet sind und pro Kunde separat gespeichert (Mandantenfähigkeit). Der Upload in die Cloud könnte durch den Kunden automatisiert werden, um den Aufwand zu senken und keine Daten zu vergessen. Auch erscheint ein längeres Zwischenspeichern in der Cloud nicht zwingend notwendig, wenn die Daten vom Kunden in die Cloud gestreamt, dort automatisch aufbereitet und dann an ein Monitoring Tool der Beratung weitergeleitet werden. Eine alternative, sehr generische Option wäre die Datenübertragung mittels einer RESTAPI. Jedes beteiligte Unternehmen würde seine Daten über eine solche Schnittstelle anbieten. Das abrufende Unternehmen (bzw. digitale Plattform) würde dann die gewünschten Daten dort zur Analyse anfordern.

In der weiteren Bearbeitung der Kundendaten wird das Beratungshaus regelmäßig prüfen, ob die Kombination mit weiteren externen oder internen Daten zu einem Mehrwert für die Analyse führt (Abb. 2). Externe Daten können beispielsweise von Markforschungsinstituten gekauft oder aus sozialen Medien extrahiert werden. Relevante interne Daten betreffen u.a. Best Practices, Lessons Learned aus früheren Kundenprojekten und spezielle branchenbezogene Hintergrundinformationen. Hier kann der Erfahrungsschatz eines Beratungsanbieters einen erheblichen Unterschied gegenüber einem reinen Anbieter analytischer Applikationen ausmachen. 


\section{Business Intelligence Gesamtlösung}

\section{Data Warehouse}

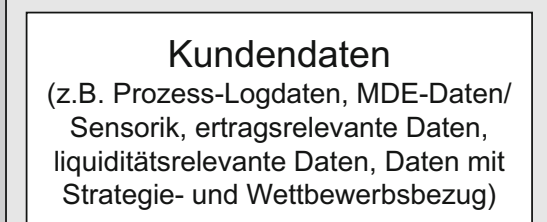

\section{Ergänzende Externe Daten}

(z.B. Geostrukturen,

Marktforschungsdaten/Trends,

Benchmarks/Branchenwerte, demografische Daten, Social Media)

\section{Interne Daten}

(z.B. anonymisierte Projektdaten,

Best Practices,

Erfahrungswerte/Branchenwissen)
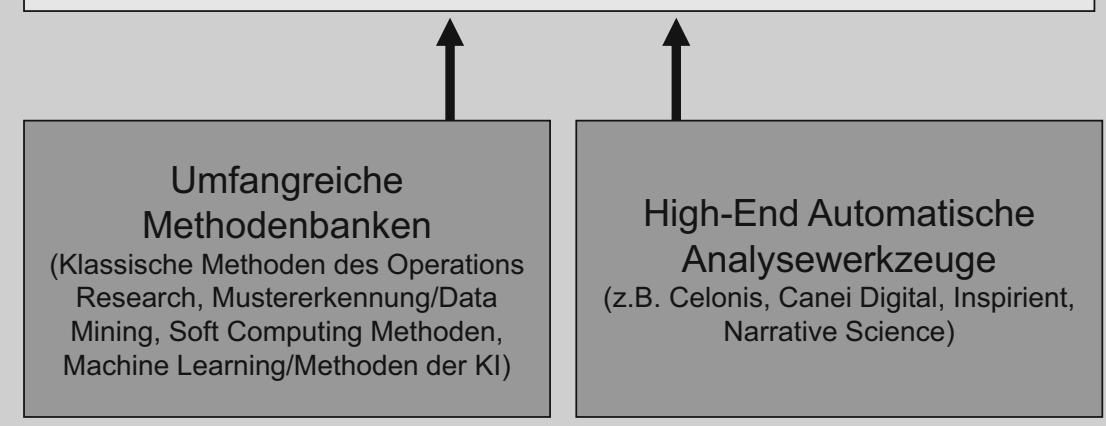

Abb. 2 Kernkomponenten der BI-Lösung des Beratungsunternehmens

Für die eigentliche Analyse der integrierten Datenbasis im Rahmen einer Business Intelligence Gesamtlösung stehen dann zwei verschiedene Ansätze zur Verfügung. Der erste Ansatz setzt auf umfangreiche Methodenbanken. Die darin enthaltenen Werkzeuge können, je nach Anwendungsfall, sowohl auf klassischen Methoden des Operations Research beruhen, als auch moderne Verfahren (Data Mining, Soft Computing, Künstliche Intelligenz) umfassen. Jede Methode müsste dabei einzeln ausgewählt und auf die gegebene Analyseproblematik abgestimmt werden (Customizing). Dies erfordert auf Seiten der Experten des Beratungshauses ein sehr hohes methodisches Wissen, verbunden mit tiefem Wissen über die Anwendungsdomäne des betreffenden Kunden. In der Praxis werden nur sehr wenige Beratungsanbieter diesem hohen Anspruch gerecht werden können, selbst unter den Großen der Branche.

Ein gänzlich anderer und voraussichtlich für die meisten Anbieter gangbarerer Weg bestände darin, High End Analysewerkzeuge einzusetzen, die weitgehend selbst entscheiden können, welche Methoden im gegebenen Einzelfall geeignet sind. Nachfolgend werden beispielhaft einige Werkzeuge vorgestellt, die bereits heute verfügbar sind und laufend weiterentwickelt werden. So bietet die Firma Celonis aus 
München eine bereits relativ bekannte Lösung für das Process Mining (v.d. Aalst 2016) an. Sie analysiert vorhandene Log-Daten von Unternehmenssoftware automatisch und liefert dann nicht nur eine Visualisierung der faktischen Prozesse im Unternehmen (Standardprozess und beliebige Abweichungen), sondern kann auch Verbesserungsvorschläge machen. Dies ist typische Beraterarbeit. Dabei kann die Celonis-Lösung schneller mehr Ergebnisse liefern als klassische Prozessberater. Einschränkend ist zu beachten, dass Process-Mining-Tools die Existenz von Logfiles voraussetzen.

Dies gilt auch für allgemeinere analytische Anwendungen, die sowohl auf Basis klassischer Statistik als auch modernen heuristischen Ansätzen und Lernverfahren arbeiten. Ein Beispiel hierfür ist die Lösung der Firma Inspirient aus Berlin (Wittenburg 2017). Hierin wird die analytische Routinearbeit von (Junior-)Strategieberatern automatisiert. Betriebswirtschaftliche Datensätze, zum Beispiel in MS-Excel, lassen sich innerhalb kürzester Zeit automatisch analysieren. Die Ergebnisse werden thematisch sortiert in Form von fertigen Präsentationsfolien erzeugt. Ein nächster Schritt in der Weiterentwicklung solcher Systeme ist die Kombination der Verfahren mit Branchenwissen, z. B. zur Bonitätsprüfung, Patentanalyse und Betrugserkennung.

\subsection{Value Network}

Neben dem Beratungshaus als Data Facilitator, seinen datenliefernden Kunden sowie anderen externen Datenquellen, den Infrastruktur- und Tool-Dienstleistern gehören noch weitere mögliche Beteiligte zum Wertschöpfungsnetzwerk (Abb. 1). Wenn beispielsweise nicht genug Berater mit den benötigten Qualifikationen verfügbar sind, wäre es denkbar, externe Experten über eine digitale Plattform in die Analyse einzubinden. Dabei könnte es sich beispielsweise um Wissenschaftler oder Mitarbeiter von Forschungsinstituten handeln, ebenso wie hoch spezialisierte freiberufliche Berater.

Es ist sogar vorstellbar, dass diese Experten regional verteilte Crowdworker mit der erforderlichen Spezialisierung sind, die auf Abruf für den Anbieter tätig werden. Betrachten wir als Beispiel die Crowdsourcing-Dienste von Kaggle. Das Geschäftsmodell von Kaggle besteht darin, Menschen mit einem Problem und den dazugehörigen Daten mit Experten auf der ganzen Welt zu verbinden, die zu den Daten passende Analysemodelle erstellen können. Die Verbindung ist 1:n, so dass für ein gegebenes Problem in Form eines Crowd-Contests eine Vielzahl unterschiedlicher Modelle auf Basis verschiedenster methodischer Ansätze entwickelt werden. Im Allgemeinen wird später nur die beste Lösung an den Auftraggeber von Kaggle verkauft. Auf diese Weise wird Data Science zu einem offenen und transparenten Wettbewerb, mit Rankings und internationaler Teambildung. Dabei findet eine Form der Gamification statt, die stark auf die Motivation von Menschen baut, bessere Ergebnisse als andere zu liefern.

Aufgrund der Sensibilität der zu übertragenen Daten benötigt der Data Facilitator ebenso Zugriff auf externe Experten für Datenschutz und Datensicherheit, soweit nicht hinreichende interne Kapazitäten dazu existieren. Gleiches gilt für externe Rechtsberatung, da insbesondere bei international verteilten Datenquellen komplexe 
Rechtssituationen angemessen berücksichtigt werden müssen, beispielsweise bei der Übertragung sensibler Daten auf Cloud-Server in den USA.

\subsection{Value Finance}

Die Dienste des Data Facilitators könnten den Kunden unregelmäßig auf Pay-perUse-Basis oder laufend gegen eine monatliche Pauschale zur Verfügung gestellt werden. Das ermöglicht dem Beratungshaus bei entsprechend vielen Kunden einen beträchtlichen Grundumsatz, der das Geschäft weniger abhängig von konjunkturellen Schwankungen macht.

Durch die externe Analyse der Kundendaten kann auch neues traditionelles Beratungsgeschäft entstehen. So könnte die Analyse beispielsweise auf die Notwendigkeit einer Prozessreorganisation beim Kunden hinweisen, woraufhin ein klassisches Beratungsprojekt aufgesetzt wird, das den analytischen Input nutzt.

Interne Daten des Beratungsunternehmens (z. B. anonymisierte Benchmarks) ließen sich ergänzend an Kunden mit eigenen Analysefähigkeiten verkaufen - das Geschäftsmodell wäre dann Data Provider. Damit würden sich weitere Erlöskanäle ergeben, wobei auch hier unterschiedliche Preismodelle denkbar sind.

\section{Erwartbare Problembereiche und Umsetzungsaspekte in der Praxis}

Bei der Umsetzung eines solchen innovativen Geschäftsmodells ist mit Schwierigkeiten und Widerständen zu rechnen. So ist die notwendige Offenlegung und Übertragung von sensiblen Kundendaten ein besonders kritischer Punkt. Datenschutz und Datensicherheit sind hier von größter Relevanz (Wiener et al. 2020). Generell können zudem regulatorische Unterschiede im Hinblick darauf bestehen, welche Daten in welcher Weise übermittelt und ausgewertet werden dürfen, je nachdem in welcher Region der Welt man sich befindet und welchem Rechtssystem man dabei unterliegt. Während diese Problematik heute schwierig erscheint, zeigen die positiven Erfahrungen mit dem Konzept Vendor-Managed-Inventory in der Logistik, dass solche Widerstände überwunden werden können. Bei VMI hat sich gezeigt, dass die Vorteile letztlich die Risiken überwiegen und in der Folge hat sich dieses Partnerkonzept in Supply Chains breit durchgesetzt. Mittelfristig ist nach Meinung des Autors auch bei den Beratungsklienten mit dem Abbau solcher Vorbehalte zu rechnen. Je öfter das Data Facilitator Geschäftsmodell bei Wettbewerbern bereits erfolgreich im Einsatz ist, umso größer wird der Druck auf „Laggards“ unter den Klienten, entsprechende Dienstleistungen ebenfalls in Anspruch zu nehmen, wenn sie bei diesen Themen auf Hilfe angewiesen sind.

Alternativ zur Übermittlung sensibler Daten vom Kunden an das Beratungshaus wäre theoretisch auch eine regelmäßige Vor-Ort-Analyse beim Kunden durch qualifizierte Berater möglich. Diese könnte allerdings nur periodisch erfolgen, womit wichtige Erkenntnisse aus Daten erst mit Zeitverzug zur Verfügung ständen. Die Akzeptanz des dargestellten Geschäftsmodells wird weiterhin stark davon abhängen, dass auf Kundenseite für die Datenbereitstellung keine zu hohen Aufwände anfallen. Ebenso wird der Kunde oft nicht bereit sein, dem Beratungsanbieter uneingeschränk- 
ten Zugriff auf seine IT-Systeme zu ermöglichen, sondern die zu analysierenden Daten entsprechend einschränken.

Für den Data Facilitator besteht ein weiteres Problem darin, hinreichend qualifizierte Berater auf dem Arbeitsmarkt zu finden. Hier wird der „War for Talents“ sich in den kommenden Jahren durch die stark wachsende Nachfrage nach solchen Fachkräften, auch aus dem Bereich großer Konzerne, weiter verschärfen. Das betrifft vor allem kleinere, weniger bekannte Beratungshäuser. Hinzu kommt die Herausforderung, das neue Geschäftsmodell auch organisatorisch abzubilden. Es dürfte sinnvoll sein, dafür eigene geschäftliche Einheiten im Beratungsunternehmen zu bilden, die möglichst wenig durch die klassischen Hierarchien gestört werden können, aber mit diesen zusammenarbeiten. Dies ist eine der Lehren aus erfolgreichen Ansätzen der technologiebasierten Virtualisierung von Dienstleistungen (Nissen 2018).

Wie erwähnt, ist es notwendig, dass die Berater mit den Klienten die Ergebnisse ihrer Analysen besprechen. Nur durch eine Interpretation und Einordnung der Analyseergebnisse, z. B. durch Erfahrungswerte aus der Branche und Benchmarks, können Beratungsunternehmen tatsächlich einen Mehrwert für ihre Klienten liefern und sich von reinen SaaS-/Analyse-Dienstleistern abheben. Vor diesem Hintergrund ist auch die beobachtete ,,algorithmic aversion“ (Dietvorst und Simmons 2015) relevant. Demzufolge stehen zahlreiche Nutzer einer völligen Automatisierung von Analyse, Schlussfolgerung und Empfehlung mittels Algorithmen skeptisch bis ablehnend gegenüber. Letztlich werden die abgeleiteten geschäftlichen Entscheidungen ja von den Klienten getroffen und verantwortet. Daher erscheinen Hybridmodelle, die virtuelles und klassisches Consulting kombinieren, notwendig, um dieses technologiebasierte Geschäftsmodell in der Praxis erfolgreich zu machen.

Ein generelles Problem bei Big Data Geschäftsmodellen sind, neben der technologischen Komplexität, gemäß der Analyse von Wiener et al. (2020) die relativ hohen initialen und laufenden Kosten der Gesamtlösung bei gleichzeitig unklaren Ertragsperspektiven. Das Data Facilitator Geschäftsmodell im Consulting wird jedoch durch verschiedene Faktoren begünstigt. Erstens ist ein inkrementeller Auf- und Ausbau nicht nur möglich, sondern unter finanziellen und Risikoaspekten auch sinnvoll. Dabei sollte zunächst mit Bestandskunden begonnen werden, deren Gegebenheiten das Beratungshaus genau kennt, und dort wiederum mit besonders wichtigen Datenquellen, bevor der Service dann im Hinblick auf die Kunden- und Datenbasis sowie das einbezogene Spektrum der Analysemethoden und Werkzeuge sukzessive erweitert wird. So bleiben Risiko und Kosten handhabbar, während gleichzeitig das „Big Picture“ im Blick behalten werden muss, getreu dem Motto „Think big. Start small." In eine ähnliche Richtung geht auch das zweite Argument, dass auf die Frage zielt, wie eine solche Lösung von Beratern mit den Klienten entwickelt wird. Hier erscheint eine agile Vorgehensweise vorteilhaft, bei der bereits in einer frühen Entwicklungsphase vorzeigbare Ergebnisse im Sinne eines ,Minimum Viable Product“" entstehen. Die damit möglichen schnellen Feedback-Schleifen verhindern, dass Dinge entwickelt werden, die aus Sicht der Kunden keinen Mehrwert erzielen. Drittens darf nicht vergessen werden, dass die Erkenntnisse aus den Analysen oft auch dazu führen werden, dass Handlungsbedarf beim Klienten entsteht, der wiederum zusätzliches klassisches Beratungsgeschäft für den Anbieter generiert und somit die Erlösseite verbessert. 
Aus vertrieblicher Sicht kann es im Consulting kaum eine bessere Konstellation geben, als regelmäßig Zugriff auf kritische Datenströme des Klienten zu haben, in denen sich Beratungsbedarf offenbaren kann. Wichtig für das Beratungsmanagement ist hier, die potenzielle Principal-Agent-Problematik zu erkennen und beherrschen. Sie besteht darin, dass Berater zu Lasten des Klienten vorrangig eigene Ziele verfolgen - z.B. Ertragsmaximierung durch zusätzliche Kundenprojekte, die für den Klienten nur wenig Mehrwert bieten. Diese Problematik gibt es im Consulting allerdings häufig. Erfahrene Berater wissen aber, dass nur zufriedene Klienten, die den Empfehlungen der Berater vertrauen (können) ihnen dauerhaften Geschäftserfolg bescheren. Sie werden daher stets darauf bedacht sein, den Kundennutzen im Fokus zu haben und insgesamt eine Win-Win-Situation zwischen Berater und Klient herzustellen. Ein auf Vertrauen basierendes partnerschaftliches Miteinander ist für den Erfolg des neuen Geschäftsmodells (wie in der Unternehmensberatung generell) unerlässlich. Allerdings müssen die Klienten verstehen, dass nicht primär eine Kostensenkung durch Outsourcing hier im Vordergrund stehen sollte, sondern Wachstumschancen durch die mögliche Verbesserung der eigenen Wettbewerbsposition.

\section{Fazit und Ausblick}

In Anbetracht des War for Talents und der typischen Ressourcensituation in KMU werden viele Unternehmen dauerhaft damit überfordert sein, die im Rahmen von Digitalisierung und Vernetzung entstehenden, rasch ansteigenden heterogenen Datenmengen sinnvoll für ihre geschäftlichen Entscheidungen auszuwerten und zu interpretieren. Nach Ansicht des Autors bietet hier das Geschäftsmodell Data Facilitator für Beratungsunternehmen große Entwicklungspotenziale. Beratungsunternehmen kennen die Besonderheiten ihrer jeweiligen Branchenschwerpunkte und Klienten genau, haben Erfahrungswerte und Benchmarks aus früheren Projekten und das Vertrauen der Kunden. Sie sind somit besser als reine SaaS-/Analyse-Dienstleister in der Lage, den Kunden einen attraktiven Mehrwert zu bieten.

Für die erfolgreiche Umsetzung kommen auf den ersten Blick vor allem große Beratungsanbieter mit hoher IT-Affinität infrage, da sie über die notwendigen Ressourcen verfügen, sowohl in personeller als auch finanzieller Hinsicht. Andererseits macht es der angesprochene inkrementelle Aufbau aber auch spezialisierten kleineren Beratungshäusern möglich in dieses Geschäftsmodell einzusteigen. Allerdings besteht nach Ansicht des Autors vor allem für First Mover die realistische Chance, das mit dem Konzept einhergehende Skalierungspotenzial zum Aufbau einer starken Marktposition zu nutzen, ähnlich wie dies bei digitalen Plattformen in anderen Branchen (z. B. im Handel) bereits beobachtet werden konnte.

Funding Open Access funding enabled and organized by Projekt DEAL.

Open Access Dieser Artikel wird unter der Creative Commons Namensnennung 4.0 International Lizenz veröffentlicht, welche die Nutzung, Vervielfältigung, Bearbeitung, Verbreitung und Wiedergabe in 
jeglichem Medium und Format erlaubt, sofern Sie den/die ursprünglichen Autor(en) und die Quelle ordnungsgemäß nennen, einen Link zur Creative Commons Lizenz beifügen und angeben, ob Änderungen vorgenommen wurden.

Die in diesem Artikel enthaltenen Bilder und sonstiges Drittmaterial unterliegen ebenfalls der genannten Creative Commons Lizenz, sofern sich aus der Abbildungslegende nichts anderes ergibt. Sofern das betreffende Material nicht unter der genannten Creative Commons Lizenz steht und die betreffende Handlung nicht nach gesetzlichen Vorschriften erlaubt ist, ist für die oben aufgeführten Weiterverwendungen des Materials die Einwilligung des jeweiligen Rechteinhabers einzuholen.

Weitere Details zur Lizenz entnehmen Sie bitte der Lizenzinformation auf http://creativecommons.org/ licenses/by/4.0/deed.de.

\section{Literatur}

van der Aalst W (2016) Process mining. Data science in action, 2. Aufl. Springer, Berlin

Al-Debei M, Avison D (2010) Developing a unified framework of the business model concept. Eur J Inf Syst 19:359-376

Ansoff HI (1975) Managing strategic surprise by response to weak signals. Calif Manage Rev 18:21-33

Dietvorst BJ, Simmons JP (2015) Algorithm aversion: people erroneously avoid algorithms after seeing them err. J Exp Psychol Gen 144(1):114-126

Fürst RA (2019) Gestaltung und Management der digitalen Transformation. Springer, Wiesbaden

Kobek W (2020) Der Umgang mit Daten will gelernt sein! https:/www.humanresourcesmanager.de/news/ data-literacy-projekt-der-umgang-mit-daten-will-gelernt-sein.html. Zugegriffen: 10. Dez. 2020

Nissen V (2007) Consulting Research - eine Einführung. In: Nissen V (Hrsg) Consulting Research. Gabler, Wiesbaden, S 3-38

Nissen V (2018) Digital transformation of the consulting industry-introduction and overview. In: Nissen V (Hrsg) Digital transformation of the consulting industry. Springer, Cham, S 1-58

Overby E (2008) Process virtualization theory and the impact of information technology. Organ Sci 19:277-291

Parakala K (2015) Global consulting and IT service providers trends - an industry perspective. Technova,

Schroeder R (2016) Big data business models: challenges and opportunities. Cogent Soc Sci. https://doi. org/10.1080/23311886.2016.1166924

Sopra Steria SE (2018) Zeit für eine neue Kultur durch Business Intelligence \& Advanced Analytics. biMA ${ }^{\circledR}-$ Studie 2017/18. Sopra Steria SE, Hamburg

Waller M, Johnson ME, Davis T (1999) Vendor-managed inventory in the retail supply chain. J Bus Logist 20:183-203

Wiener M, Saunders C, Marabelli M (2020) Big-data business models: a critical literature review and multiperspective research framework. J Inf Technol 35:66-91

Wilde T, Heß ST (2007) Forschungsmethoden der Wirtschaftsinformatik - Eine empirische Untersuchung. Wirtsch Inform 49:280-287

Wittenburg G (2017) Der werttreibende Punkt jeder Datenanalyse ist es, eine Grundlage für strategische Entscheidungen zu liefern (Interview). IM+io 2017(1):22-27 